\title{
REVISÃO DE LITERATURA SOBRE A OTIMIZAÇÃO DO PROCESSO DE USINAGEM
}

Luccas Barbosa Carneiro1; Geovana Pires Araujo Lima²; Sara Marques Oliveira de Araújo Souza ${ }^{3}$; Guilherme Oliveira de Souza ${ }^{4}$

${ }^{1}$ Universidade Federal do Recôncavo da Bahia, Rua Rui Barbosa, 710 - Centro; Cruz das Almas/Bahia; luccas.lbc@ufrb.edu.br

2 Senai CIMATEC, Avenida Orlando Gomes, 1845, Piatã; Salvador/Bahia

${ }^{3}$ Senai CIMATEC, Avenida Orlando Gomes, 1845, Piatã; Salvador/Bahia

${ }^{4}$ Senai CIMATEC, Avenida Orlando Gomes, 1845, Piatã; Salvador/Bahia

Resumo: Diante da demanda da indústria manufatureira por maior competitividade em processos de usinagem, essa pesquisa teve como objetivo fazer uma revisão de literatura sobre a otimização dos processos de usinagem, verificando os principais métodos de otimização e mapeando estatisticamente como a comunidade científica se comporta acerca desse assunto. A partir de uma busca em quatro repositórios, fazendo uso de estratégias booleanas, identificou-se que os equacionamentos de Taylor e Taylor Modificado, intervalo de máxima eficiência e otimização por sistemas computacionais são os principais métodos de otimização. Além disso, verifica-se o Estados Unidos e a Engenharia de Produção como o país e a engenharia, respectivamente, que mais possuem pesquisas nessa temática.

Palavras-Chave: Otimização; Processos; Usinagem; Ferramentas; Corte;

\section{LITERATURE REVIEW ABOUT MACHINING OPTIMIZATION PROCESS}

\begin{abstract}
Given the demand of the manufacturing industry for greater competitiveness in machining processes, this research aimed to review the literature on the optimization of machining processes, checking the main optimization methods and statistically mapping how the scientific community behaves on this subject. . From a search in four repositories, using Boolean strategies, it was found that Taylor and Modified Taylor equations, maximum efficiency range and optimization by computer systems are the main optimization methods. In addition, there is the United States and Production Engineering as the country and engineering, respectively, which have more research on this topic.
\end{abstract}

Keywords: Optimization; Process; Machining; Tools; Cutting; 


\section{INTRODUÇÃO}

O processo de usinagem se caracteriza atualmente como uma das mais relevantes inovações tecnológicas para o meio industrial, pois possibilita que a produção de artefatos metálicos, atenda as demandas de variados mercados [1]. Entretanto, para esse processo é exigido alto custo de investimento e adaptações, desde a exatidão da planicidade do solo até o nível de confiabilidade dos softwares a serem utilizados. Esses fatores devem trabalhar em conjunto paralelamente para apresentar os níveis de rendimentos e precisão solicitados pela produção [1]. $O$ processo de otimização desses fatores, relacionados a Usinagem, pode ser proposto através de algoritmos ou por processo qualitativo [2]. Além disso, os processos de usinagem devem ser otimizados, com o intuito de reduzir os custos ou incrementar a produtividade. Podendo agregar vantagem competitiva à organização, sendo fundamental no cenário globalizado atual.

Partindo do contexto supracitado, esse trabalho tem como objetivo fazer uma revisão de literatura, mapeando os métodos de otimização da vida de ferramentas de corte nos processos de usinagem e identificando estatisticamente o comportamento da comunidade científica diante desse tema.

\section{A OTIMIZAÇÃO DO PROCESSO DE USINAGEM}

A otimização do processo de usinagem é uma meta permanente nas indústrias manufatureiras, já que atualmente a globalização requer uma postura competitiva cada vez mais [3]. Já no processo de usinagem, a otimização consiste na redução de custos e de tempos de fabricação, por meio do emprego de metodologias de estratégia de corte da ferramenta ou novas tecnologias de fabricação [4]. Apesar da simples substituição do ferramental garantir bons resultados iniciais, ainda podem existir eventuais melhorias no processo, como análise dos parâmetros de corte [4]. Para que a otimização no processo de usinagem apresente resultados confiáveis e coerentes com o processo produtivo, sua análise deve ser engendrada dentro do ambiente fabril, percebendo os esforços do ambiente [3].

Pode ser utilizado uma metodologia que consiste na realização de ensaios de usinagem, usando o sistema máquina-ferramenta- peça, para determinar o tempo de vida da ferramenta, a partir daí, é possível calcular o IME. Na sequência, deve ser analisado o ambiente produtivo, escolhendo a velocidade de corte que será utilizada como referência para a otimização.

Porém, é importante destacar abordagens sobre fatores que possibilitam otimização no processo, focando em parâmetro de corte, estratégia de corte, seleção da máquina ferramenta; fornecimento e monitoramento da vida da ferramenta, entre outros [4]:

- Utilização da usinagem de titânio para analisar o impacto das condições operacionais de usinagem sobre a peça [5];

- Utilização da medição do desgaste do flanco da ferramenta, através da análise de imagem [6];

- Elaboração de um procedimento para otimizar o fornecimento de ferramentas para um grupo de máquinas [7]; 
- Desenvolvimento de uma técnica de redes neurais para otimizar o processo de usinagem com base nos seguintes objetivos: tempo de produção; custo de produção e rugosidade da superfície usinada [8];

- Realização da otimização do processo de furação com base na qualidade do furo usinado e na condição de mínimo custo [9].

Para o melhor entendimento, será apresentado importantes conceitos relativos a otimização do processo de usinagem, tais como: custo em operações de usinagem; vida da ferramenta, em que serão elucidadas as equações de Taylor e Taylor expandida, intervalo de máxima eficiência e sistemas computacionais de otimização.

\subsection{Custo em Operações de Usinagem}

Existem diferentes tipos de custos relacionados ao processo de usinagem. Pode-se dividi-lo em duas categorias: Aqueles custos decorrentes do processo (custos de ferramentas, ocupações da máquina e dos operadores), e custos envolvidos com o processo (controle da qualidade da matéria prima e mão de obra indireta) [10]. Foram considerados apenas custos diretos [4]:

$$
\mathrm{K} p=\mathrm{Kus}+\mathrm{Kum}+\mathrm{Kuf} \text {, }
$$

onde: $\mathrm{Kp}$ = custo de produção por peça $[\mathrm{R} \$$ / Peça]; Kus = custo de mão-de-obra envolvida na usinagem [R / peça]; Kum = custo da máquina-ferramenta $[\mathrm{R} \$$ / Peça]; Kuf = custo da(s) ferramenta(s) $[\mathrm{R} \$ /$ Peça $]$.

\subsection{Vida da Ferramenta}

A vida da ferramenta consiste no tempo de trabalho efetivo, excluídos os tempos passivos, de uma ferramenta, até perder sua capacidade de corte. Considerando um critério de fim de vida pré-determinado, em que alcançado este tempo, a ferramenta deve ser reafiada ou substituída [10].

Porém, definir o tempo ótimo do fim de vida de uma ferramenta, é bastante complexo, cabendo muitas vezes ao operador determinar a partir da sua experiência. O que não é um procedimento preciso, podendo provocar um grande desperdício de ferramentas. Desta forma é fundamental compreender de maneira holística os fatores que impactam na vida da ferramenta. A geometria da ferramenta também influencia sua vida, podendo ser positiva ou negativa. Sem dúvida, o fator que mais impacta na vida da ferramenta é a velocidade de corte $V_{c}$ seguindo do avanço e profundidade de usinagem. Pois, o aumento da velocidade de corte implica no aumento da troca de calor entre peça e ferramenta, e ativa ou acelera os mecanismos de desgaste como difusão, oxidação, abrasão e adesão. Desta forma, Taylor propôs, no início do século $X X$, uma função de que descreve o tempo de vida (T) em função da velocidade de corte $\left(V_{c}\right)$, ilustrada da próxima seção.

\subsubsection{Equação de Taylor}


Taylor observou a relação direta entre o tempo de vida de uma ferramenta e a velocidade de corte praticada no processo de usinagem e a formulou matematicamente,por meio da relação apresentada na equação (1).

$$
T=K \cdot v_{c}^{-x}
$$

Em que, o expoente $\mathrm{x}$ e a constante $\mathrm{K}$ são dependentes do par ferramentapeça e demais condições de corte, particular a cada caso a ser analisado. Para determinar coeficientes $\mathrm{X}$ e K, pode-se utilizar as equações (2) e (3).

$$
\begin{gathered}
x=\frac{\log \frac{T_{1}}{T_{2}}}{\log \frac{V_{2}}{V_{1}}} \\
K=T \cdot v_{c}^{x}
\end{gathered}
$$

em que: $\mathrm{X}=$ coeficiente; $\mathrm{K}=$ constante da Equação de Vida de Taylor; $\mathrm{T}$ = vida da aresta de corte [min]; $V_{c}=$ velocidade de corte $[\mathrm{m} / \mathrm{min}]$.

Embora, a velocidade de corte seja o fator predominante para a vida da ferramenta, alguns pesquisadores averiguaram o impacto das grandezas de penetração, avanço e profundidade de usinagem. Que é apresentada na próxima seção.

\subsubsection{Taylor Modificada}

A Equação de Taylor modificada considera os efeitos das grandezas de penetração, que consiste no avanço e profundidade de usinagem. E partindo do pressuposto que os fatores possuem comportamento exponencial em relação aos desgastes, a Equação de Taylor modificada pode ser definida como:

$$
v^{x} \cdot f^{y} \cdot a_{p}^{z} \cdot T=Q
$$

Em que, as constantes $x, y, z$ e $Q$ dependem do material estudado, para que seja plotado o gráfico que relaciona a velocidade de corte e a vida da ferramenta.

\subsection{Intervalo de Máxima Eficiência}

Define-se como intervalo de máxima eficiência (IME), o intervalo compreendido entre as velocidades de corte. As velocidades de mínimo custo $\left(\mathrm{V}_{c o}\right)$ e de máxima produção $\left(\mathrm{V}_{\mathrm{cm} \times \mathrm{p}}\right)$ [11]. O $\mathrm{V}_{\mathrm{cm} \times \mathrm{p}}$ possibilita a obtenção do menor tempo de fabricação $\mathrm{e}$ pode ser calculada por:

$$
V_{\text {come }}=\left\{\frac{K \cdot\left(S_{h}+S_{m}\right)}{60 \cdot(x-1) \cdot\left[K_{f t}+\left(\frac{S_{h}+S_{m}}{60}\right) \cdot t_{f t}\right]}\right\}^{\frac{1}{x}}
$$

Em, que: $t_{n}=0$ tempo de troca de ferramenta [min]. 
Figura 1 - Intervalo de máxima eficiência, Coppini (2008)

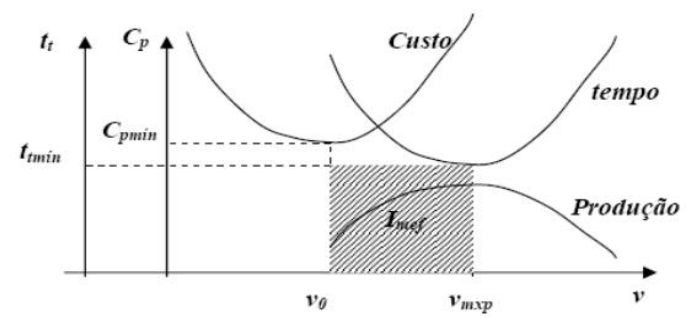

Na figura 1, é possível observar que existe outro valor de velocidade de corte dentro do IME, para o qual o tempo de confecção da peça é idêntico, porém, o custo é bem menor [10].

\subsection{Sistemas Computacionais de Otimização}

Simulações computacionais têm sido amplamente utilizadas em processos de otimização de usinagem buscando-se atingir o "modelo ótimo" em diversos aspectos: qualidade da peça final acabada, melhoria de tempo agregado de usinagem e vida útil de ferramenta. É importante destacar a necessidade de encontrar uma posição limite entre o máximo aproveitamento dos recursos da ferramenta de corte e da máquinaferramenta e o menor custo viável para a operacionalização dos processos de usinagem $[4,11]$.

Diversos trabalhos vêm apresentando aplicações de simulações computacionais na busca de otimização em processos de usinagem. Um dos trabalhos aplicou a técnica do erro quadrático médio multivariado (NBI-EQMM) sob a inserção normal à fronteira, analisando a relação custo/benefício de uma ferramenta cerâmica CC-650. Esse método obteve otimização multi objetivas, obtendo condições ótimas de custo, qualidade e produtividade [13]. Outra pesquisa, através de simulação computacional, analisou parâmetros influenciadores na definição do processo de corte, fazendo uso da abordagem de margem de contribuição. Dentre os fatores avaliados, os autores descrevem fatores de grande influência como custo de máquina e custos de ferramenta (associando a sua vida útil) [14]. Importantes pesquisas também foram desenvolvidas aliando-se simulações computacionais e a expectativa de ferramenta de corte utilizando equacionamentos de Taylor. Pode-se destacar o trabalho que fez uso de Algoritmos Genéticas e a vida de ferramenta de Taylor expandida visando a otimização de parâmetros de corte (velocidade de corte e profundidade de corte), demonstrando os custos mínimos e máximos de produção em função da vida útil da ferramenta. Como resultado, surgiram um leque de diversos materiais e situações de processo de corte [15].

\section{METODOLOGIA}

O presente trabalho foi desenvolvido conforme fluxo abaixo da figura 2: 


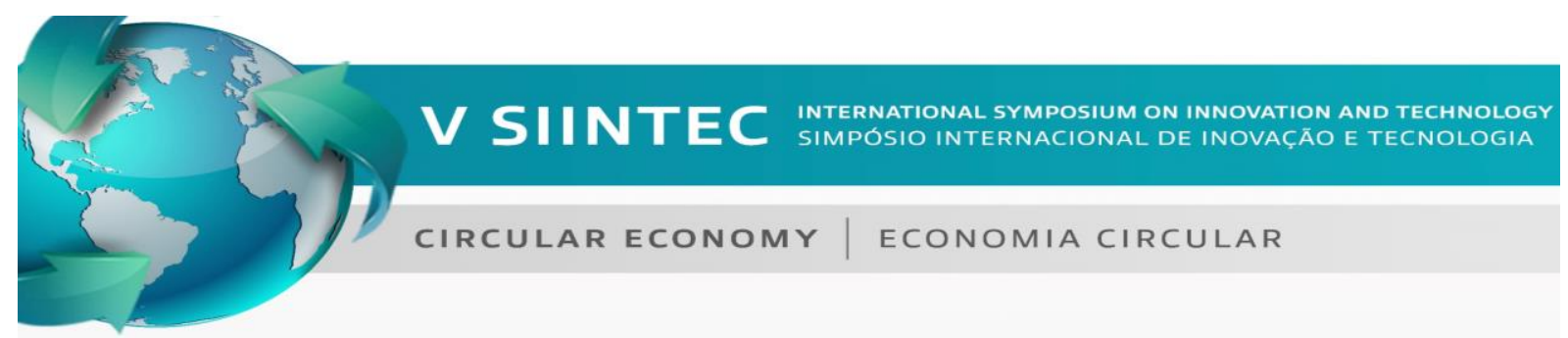

Figura 2- Fluxo de pesquisa. Desenvolvido pelos autores (2019)

\begin{tabular}{|c|c|c|c|c|c|}
\hline $\begin{array}{l}\text { DEFINIÇÃO DO } \\
\text { TEMA }\end{array}$ & $\begin{array}{l}\text { LEVANTAMENTOS } \\
\text { DOS FATORES DE } \\
\text { OTIMIZACCÄO DA } \\
\text { USINAGEM }\end{array}$ & $\begin{array}{l}\text { PESQUISA: } \\
\text { LEVANTAMENTO } \\
\text { DOS DADOS }\end{array}$ & $\begin{array}{l}\text { VALIDACCÃO DOS } \\
\text { ARTIGOS PELOO } \\
\text { ORIENTADOS }\end{array}$ & $\begin{array}{l}\text { DEFINIĆC̃OO DOS } \\
\text { TÓPICOS } \\
\text { PERTINENTES }\end{array}$ & $\begin{array}{l}\text { RELATOS DAS } \\
\text { EVIDÊNCIASE } \\
\text { CONSIDERAÇŌES } \\
\text { PERTINENTES }\end{array}$ \\
\hline
\end{tabular}

A pesquisa bibliográfica foi realizada por meio do sistema de buscas e portais periódicos: Web of Science, Science Direct, Google Acadêmico. Também foram utilizadas teses e livros em visita à biblioteca do Centro Universitário do Senai Cimatec, Bahia. A estratégia da busca consistiu nos descritores abaixo, com o uso de and, e, ou e or:

- Otimização, Processos, Usinagem, ferramentas, corte;

- Optimization, Process, Machining, Tools, Cutting.

\section{RESULTADOS E DISCUSSÃO}

A otimização da usinagem, é observada desde Taylor em 1900. E esta é uma área crítica para as organizações manufatureiras, pois afeta diretamente a sua lucratividade, seja por meio da redução dos custos ou aumento da produtividade. $E$ em um cenário de alta competitividade, espera-se que os processos sejam cada vez mais otimizados. A sustentação dessa hipótese implica no aumento da densidade de publicações. Neste sentido, foi analisado se o desenvolvimento de pesquisa nesta área está em crescimento, por meio da observação do número de publicações. A base de dados Web of Science foi selecionada, devido sua grande abrangência, e os descritores usados para busca foram: "optimization AND machining process". A quantidade de publicações por ano é apresentada no gráfico 1 (a) e os países que mais publicam em (b).

Gráfico 1: (a) Número de publicações por ano, $\quad$ (b) países que mais publicam

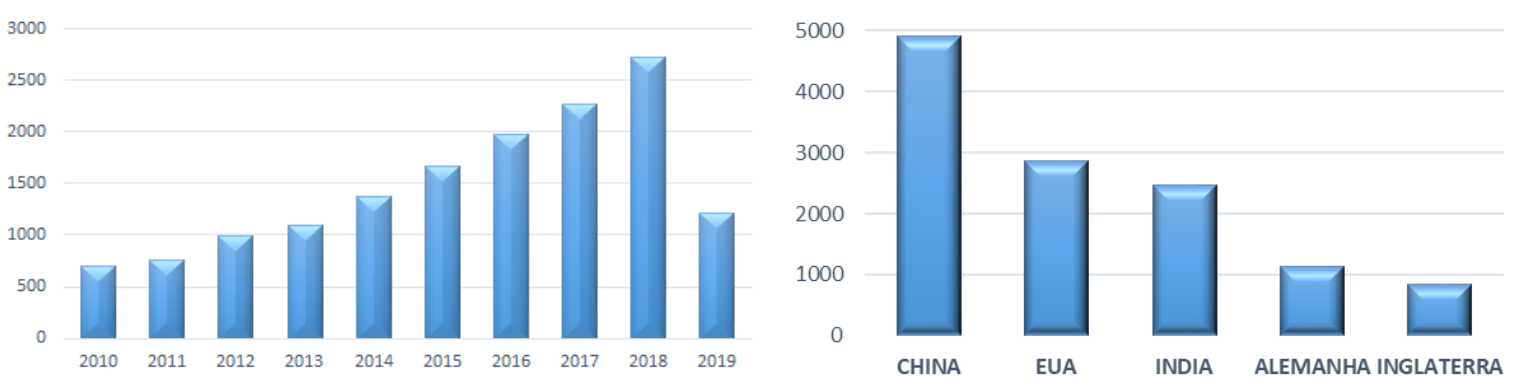

Entre as pesquisas mapeadas, as principais áreas de publicação são: Engenharia de Produção; Engenharia Elétrica e Eletrônica; Inteligência artificial e Ciência da Computação; Engenharia Mecânica; Sistemas de controle e Automação; Áreas Multidisciplinares em Ciência dos Materiais e Engenharia Industrial. O gráfico 2 ilustra a porcentagem de publicações para cada área.

Gráfico 2: Áreas das publicações
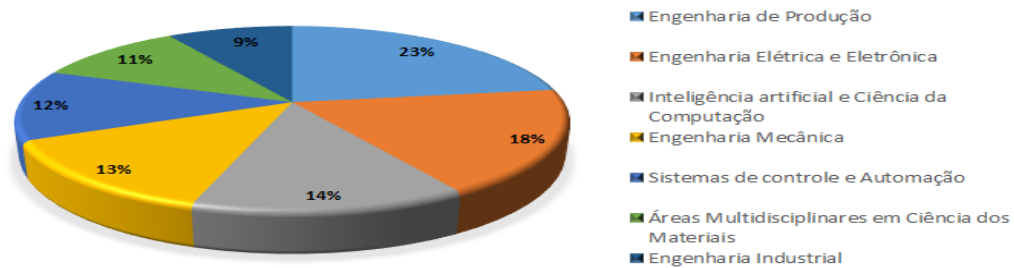
Com base nos artigos analisados, foi observado que Mukherjee [15], identificou a partir de uma revisão de literatura, as diferentes abordagens desenvolvidas para otimizar os processos de usinagem, como: Regressão Estatística; Rede Neurais; Lógica Fuzzy; Método Taguchi; Response surface design methodology; Algoritmo iterativo matemático de busca; Algoritmo Genético; Simulated annealing; Tabu search. Estas abordagens variam desde o desenvolvimento de um simples tratamento matemático até a utilização de conceitos avançados de inteligência artificial. Já Coppini e Baptista [3], concentram-se na obtenção de dados em ambiente fabril para a propor alternativas para a otimização dos parâmetros de usinagem. Partindo do pressuposto de que para realizar uma otimização de maneira confiável é imprescindível conhecer a vida útil da aresta de corte. Uma vez que muitas vezes a aresta de corte é trocada antes da sua completa utilização. A modelagem matemática foi baseada na equação de Vida de Taylor.

Para a definição dos parâmetros, a usinagem foi executada em ambiente fabril, em um número estabelecido de peças, adotando as velocidades de corte disponíveis em catálogo. Desta forma, é obtida a vida da ferramenta e o tempo de corte para cada velocidade de corte, possibilitando calcular os valores dos coeficientes supracitados. Com isso, é possível identificar um tempo ótimo de troca da ferramenta de corte. A otimização da usinagem deve ser sistematizada a partir de análise holística dos fatores que afetam os processos, levando em consideração as condições de corte e ferramentas da fábrica, dentro dos limites técnicos/econômicos.

\section{CONCLUSÃO}

O presente artigo teve como objetivo fazer uma revisão de literatura acerca dos principais métodos de otimização de usinagem e como esse tema se comporta estatisticamente no meio científico. Verificou-se que as mais recentes pesquisas no que tange a otimização de processos de usinagem no aumento da vida ferramenta de corte se dá nas seguintes "frentes científicas": equacionamento de Taylor e Taylor Modificado, intervalo de máxima eficiência e otimização por sistemas computacionais. Utilizando-se como estratégias de busca em diversos repositórios eletrônicos e físico, através de booleanos, palavras-chaves como "otimização", "processos", "Usinagem", "ferramentas", "corte" (e suas respectivas traduções em inglês), visualizou-se, através do gráfico 1 (a), uma tendência crescente de publicações de trabalhos nessa área, obtendo-se um pico de mais de 2500 trabalhos publicados no ano de 2018. Observouse que países como China e EUA detém praticamente metade das publicações a respeito de temática de otimização, de acordo com o gráfico 1 (b). No que se diz respeito a identificação das engenharias que mais tratam sobre a questão da otimização, se destacam a Engenharia de Produção, Engenharia Elétrica e Eletrônica e Inteligência artificial e Ciência da Computação (gráfico 2).

Como sugestão de futuras pesquisas, há a possibilidade de revisões de literatura somente no espaço científico brasileiro, para verificar se as pesquisas sobre otimização de ferramentas estão alinhadas com a tendência mundial.

\section{REFERÊNCIAS}

'LEITE, Y., LEITE, W., SILVA, A., SILVA, B., VELHO, L., 2018. Proposta de melhoria para aumento de produtividade em um centro de usinagem. Revista de Gestão e Tecnologia. 113 a 125. 
${ }^{2}$ HEGAB, H., DARRAS, B., KISHAWY, H. 2018. Towards sustainability assessment of machining processes. Journal of Cleaner Production, 694 a 703.

${ }^{3}$ BAPISTA. E.A., COPPINI, N. L. Otimização do Processo de Usinagem com Auxílio de sistema Especialista. Congresso Brasileiro de Engenharia de Fabricação, COBEF. Paraná, Basil, 2001.

${ }^{4}$ BAPTISTA, E. A. Desenvolvimento De Um Sistema Especialista Para A Otimização Do Processo De Usinagem Baseado Na Web, Universidade Metodista de Piracicaba, Faculdade de Engenharia Mecânica e de Produção. Programa de Pós-Graduação em Engenharia de Produção, Doutorado. 2004

${ }^{5}$ RIBEIRO, M. V.; MOREIRA, M. R. V.; FERREIRA, J. R. Optimization of titanium alloy (6A1-4V) machining. Journal of Materials Processing Technology, v. 143-144, n. 1, p. 458-463, Dec. 2003.

${ }^{6}$ SORTINO, M. Application of statistical for optical detection of tool wear. International Journal of MACHINE TOOLS \& MANUFACTURE: Design, Research and Application, v. 43, n. 5, p. 493-497, Apr. 2003.

7VRECER, G.; CUS, F. Planning of tool supply to a group of machines. Journal of Materials Processing Technology, v. 133, n. 1-2, p. 214-217, Feb. 2003.

8ZUPERL, U.; CUS, F. Optimization of cutting conditions during cutting by using neural networks. Robotics and Computer Integrated Manufacturing, v. 19, n. 1-2, p. 189-199, Feb/Apr. 2003.

${ }^{9} \mathrm{KIM}$, D.; RAMULU, M. Drilling process optimization for graphite/bismaleimidetitanium alloy stacks. COMPOSITE STRUCTURES, v. 63, n. 1, p. 101-114, Jan. 2004.

${ }^{10}$ COPPINI, N. L., MARCONDES, F.C., DINIZ, A. E. Tecnologia de Usinagem dos materiais. $7^{\circ}$ Edição, Ed. Artliber,2008.

${ }^{11}$ ROSA, A. F.C., LIBRANTZ, A.F.H., COPPINI, N.L., BAPTISTA, E. A., Simulated Annealing Technique Applied For Parameters Optimization In Machining Processes, UNINOVE, 2004;

${ }^{12}$ COSTA, Danielle Martins Duarte et al. Método nbi-eqmm para otimização do custo, qualidade e produtividade de um processo de torneamento de aço endurecido: análise custo $\mathrm{x}$ benefício de duas ferramentas de corte. In: Congresso Colombiano de costos y gestión, 2., 2015, Medellín. Anais... . Medellín: ., 2015. p. 58 - 89.

${ }^{13}$ BAPTISTA, E. A., LIBRANTZ, A. F. H., COPPINI, N. L., 2008. Analyses of the parameters influence on the price determination based on the contribution margin concept for cutting process. In: International Conference on Advanced Manufacturing Systems and Technology, 2008 :SpringerWienNewYork, 2008. vol. 1. pp. 27-34.

${ }^{14}$ Durán, O., Barrientos, R., \& Cosalter, L. A., 2007. Aplicación de algoritmos genéticos y ecuación expandida de Taylor en la obtención del Intervalo de Máxima Eficiencia. In: IV Congresso Brasileiro de Engenharia de Fabricação - COBEF, 2007

${ }^{15}$ MUKHERJEE, I.; RAY, P. K. A review of optimization techniques in metal cutting processes.Computers \& Industrial Engineering, n. 50, p. 15 - 34, 2006. 